Archives de sciences sociales des religions

141 | janvier-mars 2008

Mémoires

\title{
L'entrée de Jean Séguy dans le GSR
}

Jacques Maître

\section{(2) OpenEdition}

Journals

Édition électronique

URL : https://journals.openedition.org/assr/13112

DOI : $10.4000 /$ assr. 13112

ISSN : $1777-5825$

Éditeur

Éditions de l'EHESS

Édition imprimée

Date de publication : 1 mars 2008

Pagination : 157-158

ISBN : 978-2-7132-2189-7

ISSN : 0335-5985

Référence électronique

Jacques Maître, "L'entrée de Jean Séguy dans le GSR », Archives de sciences sociales des religions [En ligne], 141 | janvier-mars 2008, mis en ligne le 02 juillet 2008, consulté le 06 juillet 2021. URL : http:// journals.openedition.org/assr/13112; DOI : https://doi.org/10.4000/assr.13112

() Archives de sciences sociales des religions 


\section{Danièle Hervieu-Léger}

\section{In Memoriam Jean Séguy (1925-2007)}

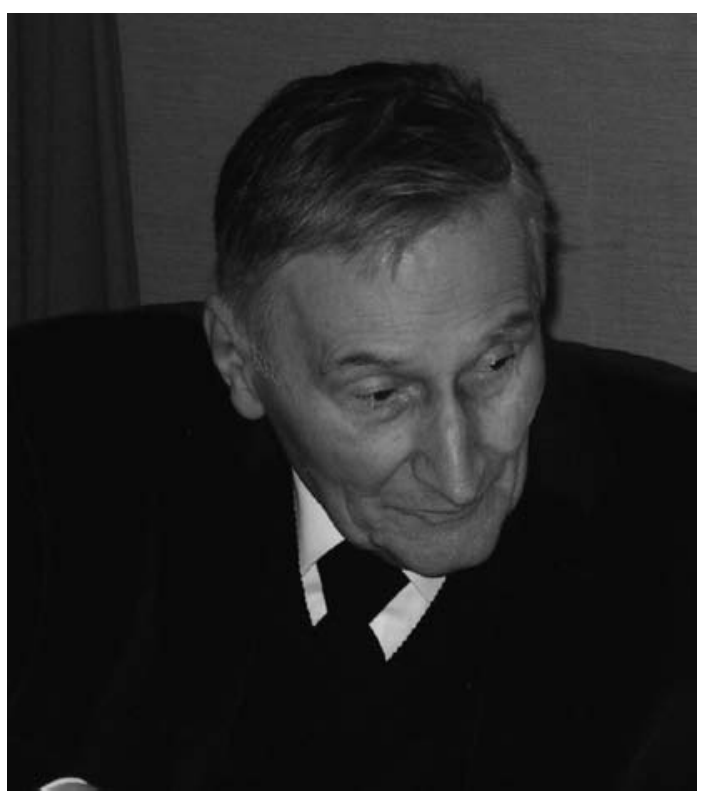

Jean Séguy nous a quittés le 9 novembre 2007, à l'âge de 82 ans. Son grand livre Les Assemblées annabaptistes-mennonites en France paru en 1977 (Mouton) le fit reconnaître comme l'un des spécialistes les plus érudits de la sociologie historique des dissidences chrétiennes des époques modernes et contemporaines. Toute sa trajectoire intellectuelle a été gouvernée par le projet de rendre compte de la puissance utopique des groupements volontaires - sectes radicales issues de la Réforme d'une part, ordres religieux d'autre part - qui réunissent des croyants qualifiés, aspirant à une vie toute entière organisée par la foi. Ce christianisme intégral est réservé à une élite de virtuoses capables de se soutenir mutuellement dans leur refus de tout compromis avec la politique et la culture mondaines. Il est au principe des expérimentations communautaires d'une alternative sociale, qui vaut anticipation du Royaume, mais qui se veut aussi interpellation du monde tel qu'il est, au prix du conflit avec les lois des États et des Églises. 
C'est auprès d'Henri Desroche - à qui alla, jusqu'à la fin de sa vie, sa reconnaissance d'avoir converti le professeur d'anglais qu'il était à la recherche en sociologie des religions - que Jean Séguy affina ses premières approches de la religion comme utopie. Entré en 1960 au Centre National de la Recherche Scientifique où il effectua toute sa carrière, c'est dans la rumination ininterrompue des œuvres de Max Weber et d'Ernst Troeltsch qu'il construisit le cadre théorique de ses analyses du christianisme comme religion de prophétie éthique. Sur toute la durée de son parcours de recherche, il affronta, de diverses façons, la question du conflit religieux. Paru en 1973, le premier livre qui le fit connaître, et qui portait sur l'œcuménisme des groupes chrétiens dissidents, tardivement réintégré dans les institutions officielles, s'intitulait déjà Les conflits $d u$ dialogue (Cerf). Le dernier ouvrage qu'il publia en 1999, et qui rassemble douze textes clés de son " parcours wébérien " porte le titre Conflit et utopie, ou réformer l'Église (Cerf).

Ce dernier livre est en fait un recueil de ses articles les plus importants, articles peaufinés longuement, avec la rigueur et la minutie érudite qui étaient sa marque propre. Cette marque, Jean Séguy - qui attachait également le plus grand prix à l'exercice serré qu'est la recension d'ouvrages - s'employa à la transmettre aux Archives, dont il fut rédacteur en chef attentif et exigeant de 1980 à 1988.

Le conflit religieux, dont sa pensée déploie les tours et retours sur différents terrains protestants et catholiques, est conflit social. Il est tout autant conflit personnel. Il traverse le croyant lui-même, écartelé entre les exigences de l'Esprit et l'obligation incontournable de réaliser sa vocation dans ce monde-ci. Et c'est en ce point, bien sûr, que se livre l'enjeu personnel qui fait aussi de ce parcours savant une trajectoire spirituelle. Cette tension qu'il explorait fut aussi sans doute la blessure, qui marqua toute la vie de Jean Séguy. Une tension que l'on peut rassembler en une question, évidemment insoluble: Comment, pour pouvoir vivre, composer avec le monde, tout en préservant en même temps cette exigence fondatrice de la radicalité chrétienne qui requiert de ne rien céder à ce qui lui est « extérieur»?

Je ne compte pas les fois où j'ai débattu avec lui, sur le mode plaisant ou sur un mode plus polémique, de la solitude inexorable à laquelle le conduisait, selon moi, cette conception redoutable, et discutable, de l'exigence chrétienne. Il avait choisi d'en payer le prix, mais je peux témoigner qu'il n'en a pas moins souffert.

Ce choix, cependant, ne fut pas seulement souffrance. Il lui valut aussi l'admiration et l'affection de tous ceux qui apprenaient à connaître, derrière l'austérité du chercheur, la générosité et la sensibilité d'un homme qui était le plus fidèle et le plus attentif des amis.

Ceux-ci étaient nombreux dans les milieux universitaires et les réseaux savants qu'il fréquenta aux différentes époques de sa carrière, à Paris, à Sherbrooke, à Oxford, à Louvain ou à Padoue. Ils étaient nombreux aussi dans les institutions religieuses dont il était familier, chez ses amis mennonites, à l'Institut catholique 
de Paris ou dans la Compagnie de Jésus, à laquelle il réserva sans doute ses attachements les plus puissants.

Le merveilleux professeur qu'il était laisse derrière lui une génération de chercheurs, en France et à l'étranger, qui ont appris leur métier auprès de lui. L'attention bienveillante qu'il accordait à chacun de ses étudiants, le souci qu'il avait de leur réussite et non de sa propre gloire à travers eux, la douceur qu'il savait leur manifester quand ils étaient dans le doute : c'est là le souvenir le plus précieux que nous, qui avons été de ceux-là, garderons de lui.

Danièle HERVIEU-LÉGER

Présidente de l'École des Hautes Études en Sciences Sociales hervieu@ehess.fr

\section{L'entrée de Jean Séguy dans le GSR}

Le noyau originel du Groupe de Sociologie des Religions et des Archives (Henri Desroche, François-André Isambert, Jacques Maître et Émile Poulat) fonctionnera tel quel pendant des dizaines d'années ; les chercheurs et techniciens qui viendront s'agréger à l'équipage prendront l'habitude d'appeler ces fondateurs "les archevêques ", terme dont l'humour souligne aussi la hiérarchisation implicite qui caractérisera le fonctionnement du laboratoire et de la revue. Pourtant, un archevêque supplémentaire se trouvera intronisé dès 1960 : Jean Séguy.

Desroche était entré au CNRS en 1951; Isambert et Maître en 1954 ; Poulat, en 1955. Je voudrais évoquer ici les circonstances dans lesquelles Séguy se présente à son tour en 1960. À cette époque, je suis membre de la Section de sociologie du Comité national de la recherche scientifique et je dois donc, à ce titre, participer à la sélection des candidats. Je me trouve ainsi destinataire d'une lettre dactylographiée de Séguy, introduisant son dossier, en date du 11 juin 1960. Le texte de ce document révèle d'emblée la façon d'être de notre collègue.

Mon curriculum vitae ne me semble pas très susceptible de vous aider à défendre ma candidature. À toutes fins utiles, voilà : licence ès Lettres (anglais !); certificat d'histoire des religions (option A, Christianisme) ; élève titulaire de l'École Pratique des Hautes Études (Protestantisme et origines chrétiennes). À part cela, enseignement du français en Angleterre (un an), et en Égypte (deux ans), puis six ans d'enseignement de l'anglais dans des établissements honorables, mais catholiques! Quant à ma production, j'en joins la liste. Il faudra, je crois, surtout insister sur ma bonne volonté !

D'avance, encore merci; et surtout ne vous mettez pas martel en tête si ça ne marche pas. Je vous demanderais simplement de me mettre un mot à la poste dès que cela vous sera possible après la session de la Commission, pour me permettre 
I 58 - ARCHIVES DE SCIENCES SOCIALES DES RELIGIONS

d'envisager l'année qui vient aussi rapidement que possible. Je fais mes envois de papier à ces Messieurs. Merci encore.

[Signé] Jean Séguy

[Ajouté à la main :] Célibataire et 35 ans (confidentiel, évidemment !!!).

Patronné par Émile-G. Léonard, ce candidat si discret compte à son actif sept publications, dont trois dans les Archives, pour la plupart en sociologie du protestantisme. Il a déjà rédigé la plus grande partie de ses 270 pages sur les Mennonites Amish des États-Unis. Les projets de recherche présentés pour le CNRS comportent la confection d'une thèse principale sur Saint Ignace et Menno Simons, et une thèse secondaire sur Les Mennonites français, avec Ernst Troeltsch comme référence théorique majeure. Sa démarche joint l'érudition historique et l'enquête de terrain ; il observe depuis cinq ans les Assemblées anabaptistes-mennonites de France. Ses textes allient une théorisation pénétrante et une écriture maîtrisée. Le « nouveau » n’est certes pas un débutant !

Aussi prendra-t-il rang archi-épiscopal dès son arrivée dans la confrérie des ASSR.

Jacques MAÎTRE jmaitre@msh-paris.fr 